\title{
Review Paper: Prevalence of VascularTrauma and Related Factors in Iran: A Systematic Review
}

\author{
Mohammad Karimian ${ }^{1,2}$, Atieh Okhli ${ }^{3}$, Abdollah Noormohammadi-Dehbalaee ${ }^{4}$, Ali Gholami ${ }^{*}$ Q, Alireza Abdi ${ }^{6}$, Ebrahim Salimi ${ }^{7}$ E, $^{2}$ \\ Milad Borji ${ }^{2,8} \mathrm{Q}$, Asma Tarjoman ${ }^{9,10} \mathrm{Q}$, Somayeh Mahdikhani ${ }^{10} \mathrm{Q}$ \\ 1. Department of Surgery, School of Medicine, Ilam University of Medical Sciences, Ilam, Iran. \\ 2. Clinical Research Development, Imam Khomeini Hospital, Ilam University of Medical Sciences, Ilam, Iran. \\ 3. Department of Nursing, Faculty of Nursing and Midwifery, Gonbad Kavoos Branch, Islamic Azad University, Gonbad Kavoos, Iran \\ 4. Departtment of Nursing, School of Allied Medical Sciences, Ilam University of Medical Sciences, Ilam, Iran. \\ 5. Department of Anesthesiology, School of Medicine, Taleghani Hospital, Kermanshah University of Medical Sciences, Kermanshah, Iran. \\ 6. Department of Nursing, Faculty of Nursing and Midwifery, Kermanshah University of Medical Sciences, Kermanshah, Iran. \\ 7. Student Research Committee, Faculty of Nursing and Midwifery, Kermanshah University of Medical Sciences, Kermanshah, Iran. \\ 8. Zoonotic Disease Research Center, Ilam University of Medical Sciences, Ilam, Iran. \\ 9. Student Research Committee, Faculty of Nursing and Midwifery, Ilam University of Medical Sciences, Ilam, Iran. \\ 10. Non-comunicable disease research center, Ilam University of Medical Sciences, Ilam, Iran. \\ 11. Department of Public Health and Infectious Diseases, School of Medicine, Sapienza University of Roma, Italy.
}

use yourdevice to scan
Trauma and Related Factors in Iran: A Systematic Review. International Journal of Medical Toxicology and Forensic Medicine.
2021; 11(2):31441. https://doi.org/10.32598/ijmtfm.v1li2.31441
def/https://doi.org/10.32598/ijmtfm.v11i2.31441

\section{() 00}

Article info:

Received: 24 Jul 2020

First Revision: 10 Aug 2020

Accepted: 08 Dec 2020

Published: 29 Jun 2021

\section{A B STRACT}

Background: Managing patients with Vascular Trauma (VT) is essential. This study aimed to determine the prevalence of VT and its related factors in Iran.

Methods: This systematic review was performed by two skilled researchers. To access all the Persian and English articles on VT and its influencing factors (from 2000 to August 2019), in addition to Google Scholar search engine, other international databases, such as PubMed/ Medline, Scopus, Embase, Cochrane Library, Science Direct, Web of Science (ISI), and domestic databases, such as Magiran, IranDoc, National Library of Iran Organization, SID, and Barakatkns were used. Data analysis was conducted by MA (CMA) software.

Results: The incidence of lower Lower Vascular Trauma (LVI) trauma was equal to 58.4 (95\%CI: 41.1-73.8) (I2=94.67, Q=112.57, $\mathrm{P}<0.001)$; the prevalence of upper LVI trauma was measured to be 31.5 (95\%CI: 17.7-49.7) (I2=94.48, $\mathrm{Q}=108.70, \mathrm{P}<0.001)$; the prevalence of penetrating trauma was calculated as 61.3 (95\% CI: 49.5-71.9); the prevalence of ulnar nerve injury equaled 9.8 (95\%CI: $2.8-28.6)$; the prevalence of radial nerve trauma was equal to 7.7 (95\% CI: 1.2-35.4); the prevalence of death cases was reported as 12.3 (95\% CI: 5.1-26.9); the prevalence of amputation rate was observed as 8.8 (95\%CI: 5.7-13.4); the prevalence of fasciotomy rate was equal to 22.2 (95\% CI: 13.2-34.5); the prevalence of complete artery cutting equaled 55.7 (95\% CI: 35.4-74.3), and the prevalence of incomplete artery cutting was measured as 25.5 (95\% CI: 12.1-45.9).

Conclusion: According to the study results, VT has led to various complications in patients; thus, it is critical to provide the necessary conditions to preserve the patient's life and prevent life-threatening complications. Such goals could be achieved by preventing this type of trauma and its related complications.

\author{
Keywords: \\ Complications, Systematic \\ review
}

\section{"Corresponding Author:}

Ali Gholami, MD.

Address: Department of Anesthesiology, School of Medicine, Taleghani Hospital, Kermanshah University of Medical Sciences, Kermanshah, Iran. Tel: +98 (930) 6051992

E-mail: gholamiali57@yahoo.com 


\section{Introduction}

rauma is considered an essential phenom$\mathbf{T}$ enon in the field of public health and among the main causes of death and disabilities [1, 2]. Trauma imposes great direct and indirect socioeconomic costs on the community [3]. Post-trauma problems include depression [4], reduced quality of life [5, 6], capabilities [7], and spiritual health [8]. Trauma can adversely affect different parts of the body and develop complications, such as head trauma $[9,10]$, dental trauma [11], spinal cord injury [12, 13], vascular trauma [14], and so on. Vascular Trauma (VT) is among the major and most dangerous traumas [13].

Trauma is among the leading causes of death; among the types of trauma, vascular injuries can be among the most important causes of patients' death $[15,16]$. The causes of VT can be attributed to blunt or penetrating; the prevalence and mechanisms of which vary across the world. The causes for their occurrence include falls, accidents, as well as knife and war injuries [17, 18]. Open vascular injuries can lead to patients' death; closed vascular injuries can lead to the amputation of patients; thus, they are very important $[19,20]$.

Managing patients with VT is crucial [21, 22]. These patients should be treated at eth soonest possible [23]. When taking remedies, in addition to time, other factors that can help prevent complications and treat the patient's conditions can be the extent of injury, the patient's previous arterial diseases, trauma to the soft tissue, and so on. Amputation occurs in cases in which ischemia is prolonged or is irreversible by physical examinations and tests $[24,25]$. Post-Trauma issues include amputation, fasciotomy, arterial injury, and even death $[26,27]$.

The prevalence of trauma types is considered as a topic of various Systematic Review (SR) and Meta-Analysis (MA) studies. In a review study, Othman et al. examined burn injuries in 12 countries; accordingly, trauma was identified as one of the causes of death [28] (Figure 1). In a review study by AzamiAghdash et al., the prevalence of dental trauma was reported as $17.5 \%$ [29]. A review study by Ning et al. indicated that the incidence of trauma-related to spinal cord injury was reported between 12.06 and 61.6 per million [30]. Another review study by Azami Aghdash et al. explored the prevalence of injury. As a result, the Mean \pm SD injury severity score was equal to $8.1 \pm 8.6$; however, this study did not specifically examine vascular injuries. Only a few related studies were reviewed in this field [31]; thus, estimating the prevalence of trauma is essential for this group of patients [32,33].

Considering the increasing prevalence of trauma and the role of VT in patients' life, it is necessary to conduct studies on VT. No SR and MA have been conducted in this field; therefore, this study aimed to determine the prevalence of VT and its related factors in Iran.

\section{Materials and Methods}

This SR study was conducted based on the PRISMA by two researchers who were skilled in SR. In case of any disagreement, the search was conducted by another member of the research team [34].

All the Persian and English articles on VT and its influencing factors (from 2000 to August 2019) were obtained by various databases, including Google Scholar search engine, and other international databases, such as PubMed/ Medline, Scopus, Embase, Cochrane Library, Science Direct, Web of Science (ISI), and domestic databases, such as Magiran (https://www.magiran.com/), IranDoc (https://irandoc.ac.ir), National Library of Iran Organization (ISI) (http://www.nlai.ir), SID (https://www.sid.ir), and (http://www.barakatkns.com) Barakatkns.

After the screening step, the articles were imported into the EndNote ${ }^{\mathrm{TM}}$ resource software and the duplicates were removed. To improve the quality of the search, the source list of the extracted articles was also searched.

Databases were searched using the following keywords: "Epidemiology, Prevalence, Trauma, Artery Injury, Vascular Trauma, Popliteal Artery Injury, Brachial Artery Injury, Vascular Surgery, Blunt Injuries, Popliteal Injuries, and Iran" (using AND \& OR operators). An example of a search strategy is as follows:

(Epidemiology [title/abstract]) OR Prevalence [title/ abstract]) AND Trauma[title/abstract]) OR Artery Injury [title/abstract]) OR Vascular Trauma [title/abstract]) OR Popliteal Artery Injury [title/abstract]) OR Brachial Artery Injury [title/abstract]) OR Vascular Surgery [title/abstract]) OR Blunt Injuries [title/abstract]) OR Popliteal Injuries [title/abstract]) AND Iran.

The study inclusion criteria based on PICO included the following [35]: population: in all papers in Iran, intervention: VT diagnosis based on diagnostic findings and/or clinical examinations, comparison: target variables, including gender, year, etc., and outcome: The results of the prevalence survey. 
The exclusion criteria included SR, MA, and qualitative studies, sample size in the overseas population of Iran, publishing papers not in Persian or English languages, and not having the full text of the articles.

A checklist, including the author's name, the year of publication, Mean \pm SD Age, place, aim, type of trauma (penetrating, blunt), the prevalence of trauma (LVI, upper LVI), the place of injury (ulnar nerve, radial nerve), the complications of trauma (death rate, amputation rate, fasciotomy rate, complete cutting of the artery, \& incomplete cutting of the artery). Data analysis was conducted by comprehensive MA (CMA) software.

\section{Results}

In this study, 610 articles were found in the primary search. The title and abstract of the extracted articles were reviewed; accordingly, 594 articles were excluded, finally, 16 articles were entered into the SR and MA. There were 1500 patients in the study and articles published between 2002 and 2015 years were included in the research. The details of the articles entered into the SR/ MA step are presented in Table 1.
According to the result, the prevalence of lower LVI trauma was measured as 58.4 (95\% CI: 41.1-73.8) (I2=94.67, Q=112.57, $\mathrm{P}<0.001)$. The prevalence of upper LVI trauma was measured as 31.5 (95\%CI: 17.7-49.7). Publication bias for lower LVI trauma was $Z=0.901$, $\mathrm{P}=0.367$; the same for upper LVI trauma was $\mathrm{Z}=0.150$, $\mathrm{P}=0.88$. The nerve injury results showed the prevalence of ulnar nerve injury to be 9.8 (95\%CI: $2.8-28.6)$ and the prevalence of radial nerve trauma as $7.7(95 \% \mathrm{CI}$ : 1.2 35.4) (Figure 2 \& 3).

The prevalence of penetrating trauma was calculated as 61.3 (95\% CI: 49.5-71.9). Moreover, the prevalence of lower LVI trauma was equal to 30.2 (95\% CI: 22.5-39.2).

According to the research findings, lower LVI trauma rate equaled 54.8 (95\%CI: 41.1-73.8), upper LVI trauma: 31.5 (95\%CI: 17.7-49.7), penetrating trauma: 61.3 (95\% CI: 49.5-71.9), blunt trauma: 30.2 (95\% CI: 22.5-39.2), ulnar nerve injury: 9.8 (95\% CI: 2.8-28.6), death rate: 12.3 (95\% CI: 5.1-26.9), amputation rate: 8.8 (95\%CI: 5.7-13.4), fasciotomy: 22.0 (95\% CI: 13.2-34.5), complete artery cutting: 55.7 (95\% CI: 35.4-74.3), and incomplete artery cutting equaled 25.5 (95\% CI: 12.1-45.9).
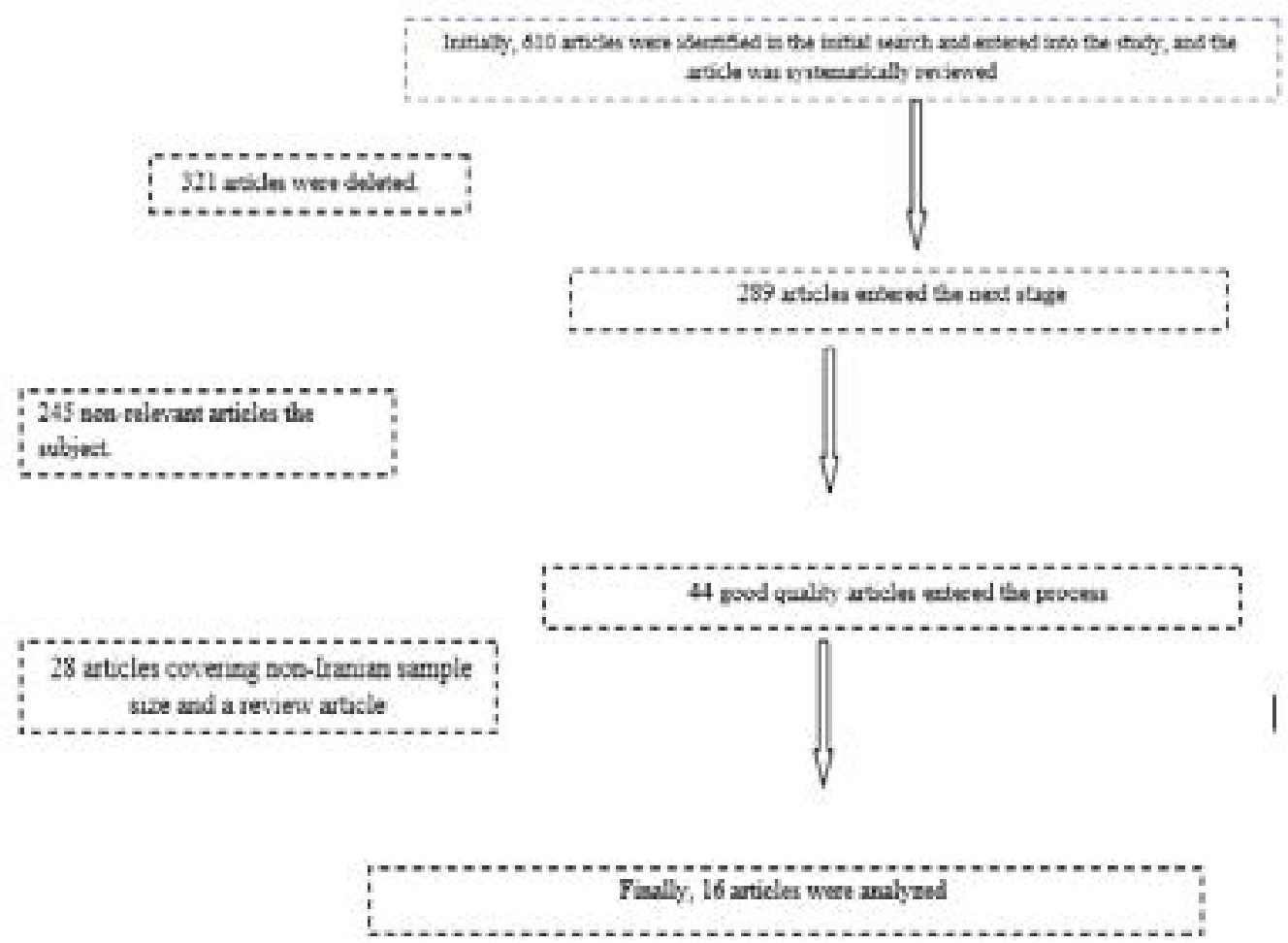

Figure 1. The flowchart of systematic review 
Table 1. Characteristics of studies entered into the SR/MAphase

\begin{tabular}{|c|c|c|c|c|c|c|c|c|c|c|c|c|c|c|c|c|}
\hline \multirow[b]{3}{*}{ zo } & \multirow[b]{3}{*}{ 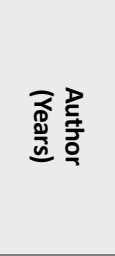 } & \multirow[b]{3}{*}{$\mathbf{z}$} & \multirow{3}{*}{ 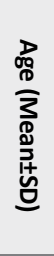 } & \multirow[b]{3}{*}{$\frac{\overline{0}}{\stackrel{0}{0}}$} & \multirow[b]{3}{*}{$\frac{2}{3}$} & \multicolumn{11}{|c|}{ No. (\%) } \\
\hline & & & & & & \multicolumn{6}{|c|}{ The Prevalence of Trauma } & \multicolumn{5}{|c|}{ The Complications of Trauma } \\
\hline & & & & & & 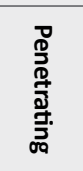 & $\frac{\text { w }}{\underline{z}}$ & 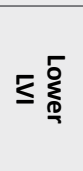 & $\begin{array}{l}\text { 등 } \\
\frac{0}{0} \\
\frac{0}{1} \\
\leq\end{array}$ & 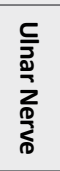 & 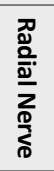 & 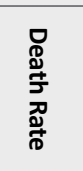 & 敢 & 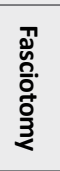 & 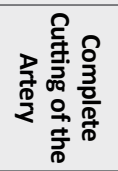 & 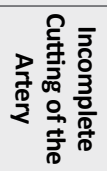 \\
\hline$\vdash$ & 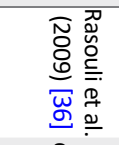 & $\overleftrightarrow{\mathrm{w}}$ & $\begin{array}{l}\underset{N}{N} \\
\stackrel{\sim}{w} \\
\stackrel{+}{+} \\
\dot{w} \\
\dot{\infty} \\
v\end{array}$ & 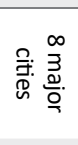 & 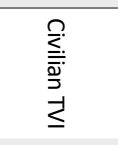 & 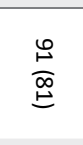 & $\begin{array}{l}\vec{b} \\
\stackrel{\widetilde{N}}{O} \\
\stackrel{\unrhd}{E}\end{array}$ & ' & ' & $\underset{\omega}{\stackrel{0}{\omega}}$ & $\stackrel{\omega}{\stackrel{\omega}{E}}$ & $\underset{\stackrel{G}{F}}{\stackrel{G}{E}}$ & $\begin{array}{l}\frac{\omega}{N} \\
\stackrel{0}{G}\end{array}$ & ' & - & ' \\
\hline$\sim$ & 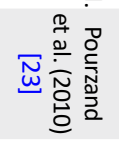 & నิ & $\stackrel{\omega}{\stackrel{\sim}{⺊}}$ & $\begin{array}{l}\frac{\vec{v}}{\bar{v}} \\
\frac{N}{.}\end{array}$ & 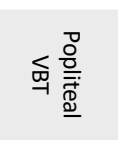 & ' & ' & ' & ' & & & $\frac{\tilde{N}}{\underline{E}}$ & $\underset{\underline{\omega}}{\stackrel{v}{\vec{\omega}}}$ & $\stackrel{a}{0}$ & , & ' \\
\hline$\omega$ & 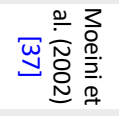 & 客 & ' & $\frac{\frac{\vec{D}}{5}}{\stackrel{9}{J}}$ & $\underline{s}$ & बे & $\underset{⿱ \mathbb{N}}{\stackrel{N}{E}}$ & ' & ' & ' & ' & $\stackrel{\omega}{\underline{\omega}}$ & ' & $\frac{6}{\omega}$ & ' & ' \\
\hline D & 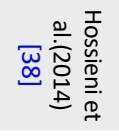 & जั & $\begin{array}{l}w \\
\stackrel{0}{ } \\
\dot{w} \\
+ \\
\dot{+} \\
\dot{0}\end{array}$ & $\frac{\vec{D}}{\stackrel{\vec{\rho}}{3}}$ & 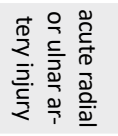 & & ' & ' & ' & $\underset{\substack{\stackrel{5}{N} \\
\stackrel{\infty}{\infty}}}{ }$ & 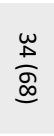 & 응 & ' & & $\begin{array}{l}\underset{\perp}{\infty} \\
\underset{\infty}{\infty}\end{array}$ & $\underset{\stackrel{b}{\sigma}}{\stackrel{\omega}{N}}$ \\
\hline$v$ & 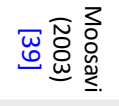 & స్తి & $\underset{\omega}{\sim}$ & $\frac{\overrightarrow{D^{\prime}}}{\frac{2}{3}}$ & $\underset{\stackrel{\Xi}{J}}{\leq}$ & $\underset{\omega}{\bar{w}} \widetilde{O}$ & $\underset{\stackrel{9}{N}}{\stackrel{N}{\mathfrak{d}}}$ & $\underset{\stackrel{w}{\omega}}{\stackrel{\sim}{\infty}}$ & $\underset{\underline{\omega}}{\stackrel{\omega}{\omega}}$ & , & ' & ' & 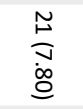 & $\underset{\substack{N \\
\stackrel{N}{N}}}{\stackrel{W}{N}}$ & ' & ' \\
\hline$\sigma$ & 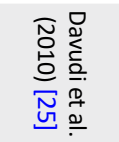 & 등 & 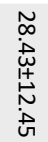 & 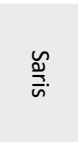 & 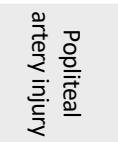 & 응 & 음 & $\begin{array}{l}\text { 음 } \\
\text { 응 }\end{array}$ & 으 & , & ' & ' & $\underset{\Xi}{\stackrel{\vec{W}}{ \pm}}$ & $\frac{N}{E}$ & ' & ' \\
\hline$v$ & 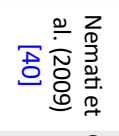 & v & $\begin{array}{l}\stackrel{\infty}{\infty} \\
\dot{\infty} \\
\infty \\
\stackrel{1}{+} \\
\stackrel{\sim}{\sim}\end{array}$ & $\frac{\vec{O}}{\frac{\vec{v}}{\bar{N}}}$ & 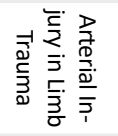 & ' & ' & ' & ' & 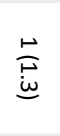 & $\stackrel{\vec{b}}{\stackrel{\vec{\omega}}{\omega}}$ & ' & ' & ' & 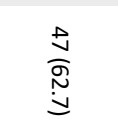 & $\underset{\substack{\infty \\
\underset{\omega}{\omega}}}{\underline{\omega}}$ \\
\hline$\infty$ & 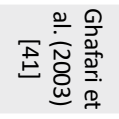 & $\vec{w}$ & ' & ' & $\leq$ & ' & ' & ' & ' & ' & $\begin{array}{l}\frac{n}{N} \\
\underline{i}\end{array}$ & ' & ' & ' & ' & ' \\
\hline 6 & 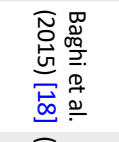 & $\infty$ & 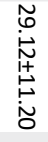 & 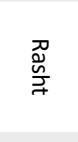 & $\underline{\leq}$ & 岕 & $\begin{array}{l}\underset{w}{w} \\
\underset{w}{\infty} \\
\dot{\infty} \\
\dot{\infty}\end{array}$ & $\underset{\substack{\infty \\
\stackrel{\infty}{w}}}{\stackrel{w}{w}}$ & $\begin{array}{l}\text { ज } \\
\text { जू } \\
\text { के }\end{array}$ & , & ' & ' & $\stackrel{0}{\underline{E}}$ & 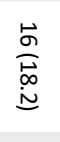 & ' & ' \\
\hline เ & 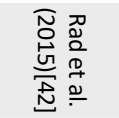 & $v$ & & 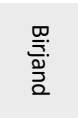 & 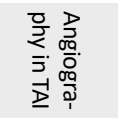 & & ' & $\begin{array}{l}\stackrel{\omega}{\infty} \\
\stackrel{\omega}{\omega}\end{array}$ & 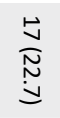 & , & ' & ' & ' & ' & ' & ' \\
\hline$\stackrel{\bullet}{\mapsto}$ & 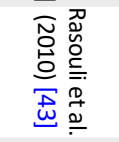 & ఝి & 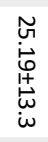 & 骨: & $\underline{\jmath}$ & $\begin{array}{l}\underset{\tilde{L}}{\tilde{W}} \\
\stackrel{\infty}{\infty}\end{array}$ & 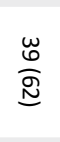 & ' & ' & ' & ' & $\frac{\omega}{\underline{N}}$ & $\begin{array}{l}\stackrel{\bullet}{N} \\
\stackrel{\omega}{G}\end{array}$ & 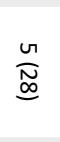 & ' & ' \\
\hline$\stackrel{\vec{N}}{ }$ & 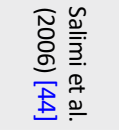 & $\underset{\omega}{\mathbb{w}}$ & $\begin{array}{l}\tilde{N} \\
\infty \\
\infty\end{array}$ & $\frac{\overrightarrow{0}}{\frac{0}{3}}$ & ¿ & 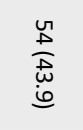 & $\begin{array}{l}\underset{b}{W} \\
\substack{w \\
\dot{\infty}}\end{array}$ & 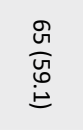 & $\underset{\substack{\omega \\
\stackrel{N}{N}}}{\underline{\omega}}$ & $\begin{array}{l}\stackrel{\mapsto}{O} \\
\stackrel{\infty}{\infty}\end{array}$ & 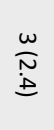 & 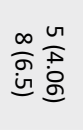 & $\underset{\stackrel{P}{\rightleftarrows}}{\stackrel{\vec{P}}{\rightleftarrows}}$ & . & ' & ' \\
\hline$\stackrel{\omega}{\omega}$ & 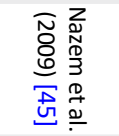 & $\stackrel{N}{N}$ & $\stackrel{\varphi}{i v}_{\stackrel{4}{v}}^{v}$ & 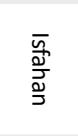 & $\underline{s}$ & 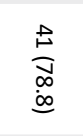 & 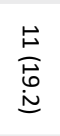 & 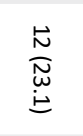 & $\begin{array}{l}\underset{\omega}{\omega} \\
\underset{\sigma}{\omega} \\
\underline{\omega}\end{array}$ & ' & ' & ' & ' & $\underset{\vec{\varphi}}{\stackrel{\bullet}{\omega}}$ & ' & ' \\
\hline 占 & 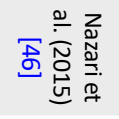 & $\$$ & 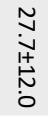 & 롫 & 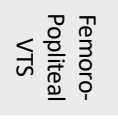 & 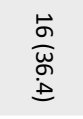 & $\begin{array}{l}\underset{\infty}{\infty} \\
\underset{0}{\omega} \\
\dot{\sigma}\end{array}$ & , & ' & & , & 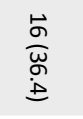 & $\stackrel{u}{\stackrel{v}{\rightleftarrows}}$ & $\begin{array}{l}\stackrel{b}{\infty} \\
\stackrel{0}{0} \\
\dot{0}\end{array}$ & . & ' \\
\hline v & 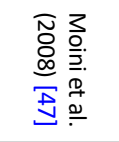 & $\approx$ & 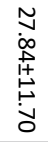 & $\frac{\frac{\vec{D}}{\mathcal{O}}}{\stackrel{9}{丂}}$ & $\begin{array}{l}\text { 罯 } \\
\end{array}$ & $\underset{\Xi}{\stackrel{N}{\Xi}}$ & 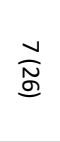 & ' & ' & ' & ' & ' & $\stackrel{\bullet}{\stackrel{\omega}{\omega}}$ & ' & ' & ' \\
\hline
\end{tabular}




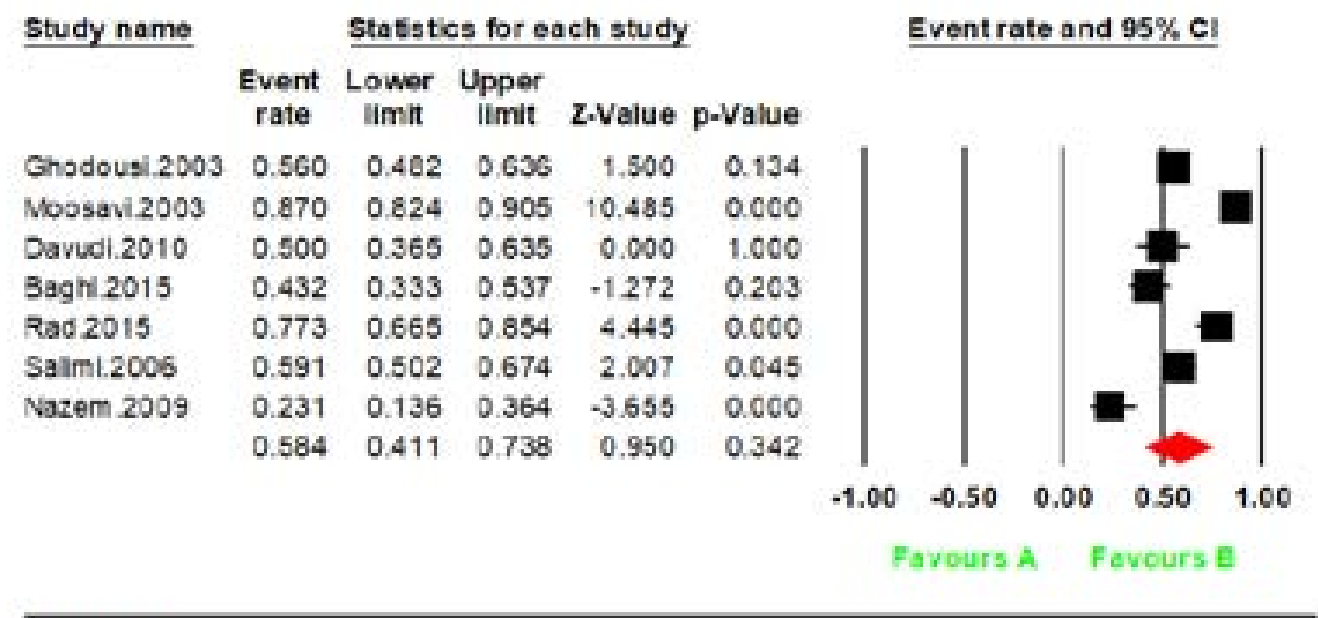

Megta Rablysis

Figure 2. The prevalence of lower LVI trauma in patients with vascular trauma International Journal of
Medical Toxicology \& Forensic Medicine

\section{Discussion}

Comparing the current study results as the first SR concerning VT and its related factors in Iran, with similar studies in other countries, is notable.

In the present study, the most common causes of trauma included accident [48-51], fall [52], and create hit [53]. Garg et al. conducted a research in India and concluded that $83.33 \%$ of patients had VT due to blunt trauma [48]. Additionally, the rate of blunt trauma in the study by Talbot et al. was measured as 3368 (57\%) [49]; the same rate equaled $57 \%$ in the study by Eslami et al. [32]; all of which were reported to be above $50 \%$. However, in a study by Ramdass et al., it accounted for $12(37 \%)$ and less than $50 \%$ of the causes of VT [50].
According to the obtained data, the incidence of death in these patients was equal to 12.3 (95\% CI: 5.1-26.9). The mortality rate in a study by Eslami et al. on 3408 patients in the pediatric vascular injuries group was measured to be $7.9 \%$ [32]. Moreover, in a study by Dhillan et al. in India, the mortality rate of vascular injuries was equal to $6.52 \%$ [51]. Furthermore, in a cohort and retrospective study by Sharrock et al., this rate was equal to $10 \%$ [52], i.e., approximately the same as the current research results. However, according to Talbot et al., 5858 patients with vascular injuries had a mortality rate of 695 $(21 \%)$, and the mortality rate of penetrating injuries was equal to $727(31 \%)$ [49]. In a study by Prichayudh et al., the rate of mortality of 55 patients with abdominal vascular injuries was calculated as 40 (59\%), i.e., greater than the rate mentioned in our study.

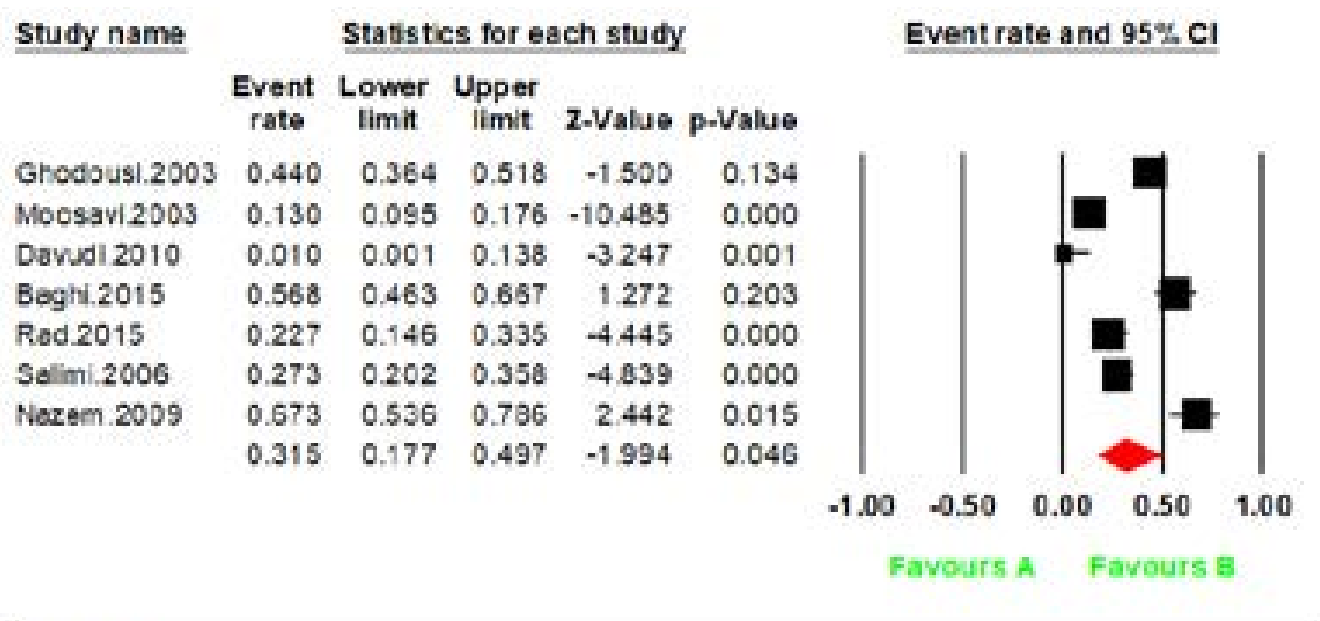

Mets Analysis.

Figure 3. The prevalence of upper LVI trauma in patients with vascular trauma

International Journal of Medical Toxicology \& Forensic Medicine 
Furthermore, the rate of amputation was measured to be 8.8 (95\%CI: 5.7-13.4). Garg et al. in India reported the rate of amputation of patients as $15(25 \%)$ cases [48]. Besides, a study by Ramdass et al. in India included 9 $(28 \%)$ cases [50]. A study by Dhillan et al. included 3 $(8.33 \%)$ cases [51]. However, in a study by Van Dongen et al. in the Afghan war injury group, the initial amputation rate was reported as $77.4 \%$ [53], which can be among the causes of trauma. Moreover, in a study by Van Dongen et al. [53] war injuries were studied, while all patients with different causes of VT were included in this study.

Failure to study the prevalence of vascular trauma based on geographical area, due to lack of geographical variation in studies was a limitation of this research. Besides, overlooking the investigation of VT based on gender, occupation, and socioeconomic status was another limitation of this study.

\section{Conclusion}

According to the present study results, VT has led to various complications in patients; thus, it is crucial to provide the necessary conditions to preserve the patient's life and prevent life-threatening complications. Such goals could be achieved by preventing this type of trauma and its complications.

\section{Ethical Considerations}

\section{Compliance with ethical guidelines}

This study was approved by the Ethics Committee of the he Student Research Committee, Kermanshah University of Medical Sciences (Code: IR.KUMS.REC.1398.811).

\section{Funding}

This research was supported by the research project (No. 3009014) Funded by the University of Kermanshah University of Medical Sciences.

\section{Author's contributions}

All authors equally contributed to preparing this article.

\section{Conflict of interest}

The authors declared no conflicts of interest.

\section{Acknowledgements}

We would like to thank the Student Research Committee, Kermanshah University of Medical Sciences, Kermanshah, Iran.

\section{References}

[1] Hemmati E, Ghayoumi-Anaraki Z, Ehsaei MR, Ghasisin L. Effect of frontal lobe traumatic brain injury on sentence comprehension and working memory. Trauma Mon. 2019; 24(1):e74353. [DOI:10.5812/traumamon.74353]

[2] Sarkarat F, Kalantar-Motamedi MH, Mahaseni-Aghdam H, Rastegarmoghadamshalduzi H. Evaluation of oral and maxillofacial trauma in Tehran from 2008 to 2016. Trauma Mon. 2019; 24(2):1-5. [DOI:10.5812/TRAUMAMON.67802]

[3] Zamani M, Esmailian M, Mirazimi MS, Ebrahimian M, Golshani K. [Cause and final outcome of trauma in patients referred to the emergency department; A cross sectional study (Persian)]. Iran J Emerg Med. 2014; 1(1):22-7. https:// www.magiran.com/paper/1563509?lang=en

[4] Blakey SM, Yi JY, Calhoun PS, Beckham JC, Elbogen EB. Why do trauma survivors become depressed? Testing the behavioral model of depression in a nationally representative sample. Psychiatry Res. 2019; 272:587-94. [DOI:10.1016/j.psychres.2018.12.150] [PMID]

[5] Kruithof N, Haagsma JA, Karabatzakis M, Cnossen MC, de Munter L, van de Ree CLP, et al. Validation and reliability of the abbreviated World Health Organization Quality of Life Instrument (WHOQOL-BREF) in the hospitalized trauma population. Injury. 2018; 49(10):1796-804. [DOI:10.1016/j.injury.2018.08.016] [PMID]

[6] Khajavikhan J, Vasigh A, Khani A, Jaafarpour M, Kokhazade T. Outcome and predicting factor following severe traumatic brain injury: A retrospective cross-sectional study. J Clin Diagn Res. 2016; 10(2):PC16-9. [DOI:10.7860/ JCDR/2016/16390.7294] [PMID] [PMCID]

[7] Carrie C, Guemmar Y, Cottenceau V, de Molliens L, Petit L, Sztark F, et al. Long-term disability after blunt chest trauma: Don't miss chronic neuropathic pain! Injury. 2019; 50(1):113-8. [DOI:10.1016/j.injury.2018.10.023] [PMID]

[8] Smothers ZPW, Koenig HG. Spiritual interventions in veterans with PTSD: A systematic review. J Relig Health. 2018; 57(5):2033-48. [DOI:10.1007/s10943-018-0680-5] [PMID]

[9] Chee JN, Hawley C, Charlton JL, Marshall S, Gillespie I, Koppel S, et al. Risk of motor vehicle collision or driving impairment after traumatic brain injury: A collaborative international systematic review and meta-analysis. J Head Trauma Rehabil. 2019; 34(1):E27-38. [DOI:10.1097/ HTR.0000000000000400] [PMID]

[10] Rahmani A, Hatefi M, Dastjerdi MM, Zare M, Imani A, Shirazi D. Correlation between serum homocysteine levels and outcome of patients with severe traumatic brain injury. World Neurosurg. 2016; 87:507-15. [DOI:10.1016/j. wneu.2015.09.016] [PMID] 
[11] Magno MB, Neves AB, Ferreira DM, Pithon MM, Maia LC. The relationship of previous dental trauma with new cases of dental trauma. A systematic review and meta-analysis. Dent Traumatol. 2019; 35(1):3-14. [DOI:10.1111/edt.12449] [PMID]

[12] Hassannejad Z, Yousefifard M, Azizi Y, Zadegan SA, Sajadi $\mathrm{K}$, Sharif-Alhoseini $\mathrm{M}$, et al. Axonal degeneration and demyelination following traumatic spinal cord injury: A systematic review and meta-analysis. J Chem Neuroanat. 2019; 97:9-22. [DOI: 10.1016/j.jchemneu.2019.01.009] [PMID]

[13] Hatefi M, Ahmadi MRH, Rahmani A, Dastjerdi MM, Asadollahi $\mathrm{K}$. Effects of curcumin on bone loss and biochemical markers of bone turnover in patients with spinal cord injury. World Neurosurg. 2018; 114:e785-91. [DOI:10.1016/j. wneu.2018.03.081] [PMID]

[14] Debette S, Schilling S, Duperron MG, Larsson SC, Markus HS. Clinical significance of magnetic resonance imaging markers of vascular brain injury: A systematic review and meta-analysis. JAMA Neurol. 2019; 76(1):81-94. [DOI:10.1001/ jamaneurol.2018.3122] [PMID] [PMCID]

[15] Gilbert F, Schneemann C, Scholz CJ, Kickuth R, Meffert $\mathrm{RH}$, Wildenauer R, et al. Clinical implications of fractureassociated vascular damage in extremity and pelvic trauma. BMC Musculoskelet Disord. 2018; 19(1):404. [DOI:10.1186/ s12891-018-2333-y] [PMID] [PMCID]

[16] Yadollahi M, Arabi AH, Mahmoudi A, Zamani M, Farahmand $\mathrm{M}$. Blunt thoracic injury mortality and clinical presentation. Trauma Mon. 2018; 23(4):e13079. [DOI:10.5812/traumamon.13079]

[17] Agrawal V, Bedi VS, Anand V, Garg MK. Management of vascular injury in counter insurgency area: A singlecenter study. Indian J Vasc Endovasc Surg. 2018; 5(1):29-34. [DOI:10.4103/ijves.ijves_65_17]

[18] Baghi I, Herfatkar MR, Shokrgozar L, Poor-Rasuli Z, Aghajani F. Assessment of vascular injuries and reconstruction. Trauma Mon. 2015; 20(4):e30469 [DOI:10.5812/traumamon.30469] [PMID] [PMCID]

[19] Gümbel D, Naundorf M, Napp M, Ekkernkamp A, Seifert J. [Diagnosis and management of peripheral vascular injuries (German)]. Unfallchirurg. 2014; 117(5):445-59. [DOI:10.1007/ s00113-014-2560-0] [PMID]

[20] Jaha L, Andreevska T, Rudari H, Ademi B, Ismaili-Jaha V. A decade of civilian vascular trauma in Kosovo. World J Emerg Surg. 2012; 7(1):24. [DOI:10.1186/1749-7922-7-24] [PMID] [PMCID]

[21] Moini M, Takyar MA, Rasouli MR. Revascularisation later than $24 \mathrm{~h}$ after popliteal artery trauma: Is it worthwhile? Injury. 2007; 38(9):1098-101. [DOI:10.1016/j.injury.2007.05.001] [PMID]

[22] Khoshnevis J, Lotfollahzadeh S, Sobhiyeh MR, Najd Sepas $\mathrm{H}$, Abbas Nejad M, Rahbari A, et al. Ruptured aneurysm of the splenic artery: A rare cause of abdominal pain after blunt trauma. Trauma Mon. 2013; 18(1):46-9. [DOI:10.5812/traumamon.8271] [PMID] [PMCID]

[23] Pourzand A, Fakhri BA, Azhough R, Hassanzadeh MA Hashemzadeh S, Bayat AM. Management of high-risk popliteal vascular blunt trauma: Clinical experience with 62 cases. Vasc Health Risk Manag. 2010; 6:613-8. [DOI:10.2147/VHRM. S11733] [PMID] [PMCID]
[24] Todd R, Vogel M, Moral H. Injuries to the peripheral blood vessels, chapter 17, ACS Surgery. New York: WebMD Professional Publishing; 2007.

[25] Davudi M, Farsavian H, Shayesteh Azar M, Tayebi P. [Evaluation of long-term outcomes of popliteal artery injury following lower extremity blunt trauma in Sari Imam Khomeini teaching hospital from 2004 to 2009 (Persian)]. J Mazand Univ Med Sci. 2010; 20(79):31-8. http://jmums.mazums.ac.ir/article-1-634-en.htm

[26] Hatz BA, Frima H, Sommer C. Selective fasciotomy for acute traumatic lower leg compartment syndrome: Is it feasible? Arch Orthop Trauma Surg. 2019; 139(12):1755-62. [DOI:10.1007/s00402-019-03260-1] [PMID]

[27] Noblet T, Lineham B, Wiper J, Harwood P. Amputation in trauma-how to achieve a good result from lower extremity amputation irrespective of the level. Curr Trauma Rep. 2019; 5:69-78. [DOI:10.1007/s40719-019-0159-1]

[28] Othman N, Kendrick D. Epidemiology of burn injuries in the East Mediterranean region: A systematic review. BMC Public Health. 2010; 10:83. [DOI:10.1186/1471-2458-10-83] [PMID] [PMCID]

[29] Azami-Aghdash S, Azar FE, Azar FP, Rezapour A, MoradiJoo M, Moosavi A, et al. Prevalence, etiology, and types of dental trauma in children and adolescents: Systematic review and meta-analysis. Med J Islam Repub Iran. 2015; 29(4):234. [PMID] [PMCID]

[30] Ning GZ, Wu Q, Li YL, Feng SQ. Epidemiology of traumatic spinal cord injury in Asia: A systematic review. J Spinal Cord Med. 2012; 35(4):229-39. [DOI:10.1179/204577231 2Y.0000000021] [PMID] [PMCID]

[31] Azami-Aghdash S, Sadeghi-Bazargani H, Shabaninejad H, Gorji HA. Injury epidemiology in Iran: A systematic review. J Inj Violence Res. 2017; 9(1):27-40. [DOI: 10.5249/jivr.v9i1.852] [PMID] [PMCID]

[32] Eslami MH, Saadeddin ZM, Rybin DV, Avgerinos ED, Eslami PW, Siracuse JJ, et al. Trends and outcomes of pediatric vascular injuries in the united states: An analysis of the national trauma data bank. Ann Vasc Surg. 2019; 56:52-61. [DOI:10.1016/j.avsg.2018.09.006] [PMID]

[33] Johnson JP, Kleiner J, Klinge SA, McClure PK, Hayda RA, Born CT. Increased incidence of vascular injury in obese patients with knee dislocations. J Orthop Trauma. 2018; 32(2):82-7. [DOI:10.1097/BOT.0000000000001027] [PMID]

[34] Moher D, Shamseer L, Clarke M, Ghersi D, Liberati A, Petticrew M, et al. Preferred Reporting Items for Systematic review and Meta-Analysis Protocols (PRISMA-P) 2015 statement. Syst Rev. 2015; 4(1):1. [DOI:10.1186/2046-4053-4-1] [PMID] [PMCID]

[35] Richardson WS, Wilson MC, Nishikawa J, Hayward RS. The well-built clinical question: A key to evidence-based decisions. ACP J Club. 1995; 123(3):A12-3. [PMID]

[36] Rasouli MR, Moini M, Khaji A. Civilian traumatic vascular injuries of the upper extremity: Report of the Iranian national trauma project. Ann Thorac Cardiovasc Surg. 2009; 15(6):389-93. [PMID]

[37] Moeini M, Khorvash B, Monnajem Zadeh M. [Study of factors effects on Morbidity of patients with vascular trauma in Sina hospital 1994 -1999 (Persian)]. Tehran Univ Med J. 2002; 60(1):15-8. https:// tumj.tums.ac.ir/article-1-1275-en.html 
[38] Hossieni-Kasnavieh SM, Chahardoli M, Basir Ghafouri $\mathrm{H}$, Sedghi A, Ramim T. [Management of acute radial or ulnar artery injury in hospital emergency department (Persian)]. SJKU. 2014; 19(4):20-6. http://sjku.muk.ac.ir/article1-1596-en.html

[39] Moosavi SR. [The effects of Time Interval on Vascular Trauma (Persian)]. J Mil Med. 2003; 5(2):99-103. http://militarymedj.ir/article-1-176-en.html

[40] Nemati M, Nosratinia H, Asefi S. [Angiographic findings of arterial injuries in extremity trauma (Persian)]. Horizon Med Sci. 2009; 15(1):15-9. http://hms.gmu.ac.ir/article1-521-en.html

[41] Ghafari A, Haidari S. [Analysis of radiologocal data related to vasocirculatory disorder in injured soldier admitted to Shahid Mustafa Khomeini hospital (Persian)]. J Mil Med. 2003; 4(4):243-6. https://www.sid.ir/en/journal/ViewPaper.aspx?id=27669

[42] Pezeshki-Rad M, Mohammadifard M, Ravari H, Farrokh D, Ansaripour E, Saremi E. Comparing color Doppler ultrasonography and angiography to assess traumatic arterial injuries of the extremities. Iran J Radiol. 2015; 12(1):e14258. [DOI:10.5812/iranjradiol.14258] [PMID] [PMCID]

[43] Rasouli MR, Moini M, Khaji A, Heidari P, Anvari A. Traumatic vascular injuries of the lower extremity: Report of the Iranian National Trauma Project. Ulus Travma Acil Cerrahi Derg. 2010; 16(4):308-12. [PMID]

[44] Salimi J, Zarei MKMR. Vascular injuries in Tehran: A review of 123 cases. Acta Med Iranica. 2006; 44(5):333-40. https://acta.tums.ac.ir/index.php/acta/article/view/3210

[45] Nazem M, Beigi AA, Sadeghi AMM, Masoudpour H. Non iatrogenic paediatric vascular trauma of the extremities and neck. Afr J Paediatr Surg. 2009; 6(1):35-9. [DOI:10.4103/01896725.48574] [PMID]

[46] Nazari I, Mashhadi M, Alamshah SM, Moosavi SM,. Outcomes of femoro-popliteal vascular trauma surgery: A threeyear survey (2011-2013) in Ahvaz Golestan Hospital (Iran). Biosci Biotech Res Asia. 2015; 12(3):2193-9. http://www. biotech-asia.org/vol12no3/outcomes-of-femoro-poplitealvascular-trauma-surgery-a-three-year-survey-2011-2013-inahvaz-golestan-hospital-iran/

[47] Moini M, Hamedani K, Rasouli MR, Nouri M. Outcome of delayed brachial artery repair in patients with traumatic brachial artery injury: Prospective study. Int J Surg. 2008; 6(1):20-2 [DOI:10.1016/j.ijsu.2007.11.005] [PMID]

[48] Garg MK, Bedi VS, Yadav A, Agarwal S, Satwik A, Srivastava A. Study of risk factors affecting the limb salvage in patients with lower extremity arterial trauma. Indian J Vasc Endovasc Surg. 2019; 6(2):115-20. [DOI:10.4103/ijves.ijves_80_18]

[49] Talbot E, Evans S, Hellenthal N, Monie D, Campbell P Cooper S. Abdominal and pelvic vascular injury: A national trauma data bank study. Am Surg. 2019; 85(3):292-3. [DOI:10 1177/000313481908500335] [PMID]

[50] Ramdass MJ, Muddeen A, Harnarayan P, Spence R, Milne D. Risk factors associated with amputation in civilian popliteal artery trauma. Injury. 2018; 49(6):1188-92. [DOI:10.1016/j.injury.2018.04.028] [PMID]
[51] Dhillan R, Bhalla A, Jha SK, Singh H, Arora A. Vascular injuries due to penetrating missile trauma in anti-terrorism ops J Trauma Inj. 2019; 32(2):93-100. [DOI:10.20408/jti.2018.032]

[52] Sharrock AE, Remick KN, Midwinter MJ, Rickard RF. Combat vascular injury: Influence of mechanism of injury on outcome. Injury. 2019; 50(1):125-30. [DOI:10.1016/j.injury.2018.06.037] [PMID]

[53] Van Dongen TTCF, Idenburg FJ, Tan ECTH, Rasmussen TE, Hamming JF, Leenen LPH, et al. Combat related vascular injuries: Dutch experiences from a role 2 MTF in Afghanistan Injury. 2016; 47(1):94-8. [DOI:10.1016/j.injury.2015.08.030] [PMID] 